\title{
Cardiogenic Shock
}

\author{
Kristen A. Smith ${ }^{1}$ and Michael T. Bigham ${ }^{*}, 2$ \\ ${ }^{I}$ Department of Pediatrics, ${ }^{2}$ Division of Critical Care Medicine, Akron Children's Hospital Department of Critical Care, \\ USA
}

\begin{abstract}
The primary function of the cardiovascular system is to provide oxygen and energy substrates to the body's tissues. In all forms of shock, there is metabolic failure - a downstream consequence of an inability to meet the metabolic demands of the tissue. In cardiogenic shock, the primary derangement leading to unmet tissue metabolic demands is cardiac dysfunction. Though cardiac impairment may be implicated in other categories of shock, primary cardiac failure in the form of cardiogenic shock is the third most common type of shock in pediatrics - trailing septic shock and hypovolemic shock. Myocardial malfunction, in other forms of shock, is secondary to ischemia, acidosis, drugs, toxins, or direct myocardial depressants due to inflammation. Cardiogenic shock, a low-output state, is characterized by elevated ventricular filling pressures, low cardiac output, systemic hypotension, and evidence of end-organ hypoperfusion. Herein, we will highlight the pathophysiology of cardiogenic shock, the various causative etiologies, the multitude of laboratory or imaging diagnostic options, current advances in therapies, and outcomes in patients.
\end{abstract}

Keywords: Pediatric cardiogenic shock, cardiac transplant, congenital heart disease, congestive cardiac failure, extracorporeal membrane oxygenation, myocarditis, ventricular assist device.

\section{INTRODUCTION}

The primary function of the cardiovascular system is to provide oxygen and energy substrates to the body's tissues. In all forms of shock, there is metabolic failure - a downstream consequence of an inability to meet the metabolic demands of the tissue. In cardiogenic shock, the primary derangement leading to unmet tissue metabolic demands is cardiac dysfunction. Though cardiac impairment may be implicated in other categories of shock, primary cardiac failure in the form of cardiogenic shock is the third most common type of shock in pediatrics - trailing septic shock and hypovolemic shock. Myocardial malfunction, in other forms of shock, is secondary to ischemia, acidosis, drugs, toxins, or direct myocardial depression due to inflammation $[1,2]$. Cardiogenic shock, a low-output state, is characterized by elevated ventricular filling pressures, low cardiac output, systemic hypotension, and evidence of endorgan hypoperfusion. Herein, we will highlight the pathophysiology of cardiogenic shock, the various causative etiologies, the multitude of laboratory or imaging diagnostic options, current advances in therapies, and outcomes in patients.

This review focuses on pediatric cardiogenic shock, excluding the neonatal period. For the latter, the readers are referred to excellent reviews on this topic $[3,4]$.

\section{PATHOPHYSIOLOGY}

Cardiogenic shock results from an impairment of cardiac output (CO), elevated systemic vascular resistance (SVR), or

*Address correspondence to this author at the Akron Children's Hospital, Division of Critical Care Medicine, One Perkins Square, Akron, OH 443081062, USA; Tel: (330) 543-8639; Fax: (330) 543-8136;

E-mail: mbigham@chmca.org both. Recall that cardiac output is equal to the product of heart rate $(\mathrm{HR})$ and stroke volume $(\mathrm{SV})(\mathrm{Eq} 1)$. Stroke volume is influenced by contractility and left ventricular filling pressure. In infants and children, cardiac output is primarily driven by heart rate due to a lack of ventricular muscle mass and thus a lack of improved contractility. Elevations in SVR hinder left ventricular ejection through increased afterload [5].

$$
\mathrm{CO}=\mathrm{HR} \times \mathrm{SV}
$$

There are four major determinants of ventricular function: contractility, heart rate, preload, and afterload. The Frank-Starling relationship is a series of curves detailing the relationship between preload and ventricular function (Fig. 1). As preload increases, so then must cardiac output. At the point when the myocytes are stretched beyond their ability to generate increased force, ventricular function worsens. At the extremes of impaired ventricular function, the heart failure may result in cardiogenic shock. In the normal heart, intracellular calcium shifts determine contractility. In the case of decreased myocardial performance, calcium handling is abnormal, leading to both systolic and diastolic dysfunction.

Compensatory responses that protect the body in other forms of shock can contribute to worsening of heart failure by further depressing cardiac function. The body naturally responds to a low output state by increasing systemic vascular resistance. However, this increase in systemic vascular resistance imparts an increase in ventricular afterload, adding to increased cardiac work and further decreasing function. In an effort to increase blood flow to end-organs, the renin-angiotensin II-aldosterone pathway is activated, thereby encouraging renal juxtaglomerular cells to increase reabsorption of water and salt. This process further 
increases preload and in a cardiogenic shock state, contributes to pulmonary and peripheral edema via excessive ventricular end diastolic volume. Another effect of decreased cardiac output is the activation of the sympathetic nervous system, releasing catecholamines. The immediate effect of this in early shock is to increase heart rate, thereby increasing cardiac output. In the long-term, however, the vasoconstrictive properties of endogenous catecholamines increase afterload, thereby contributing to heart failure and further organ dysfunction.

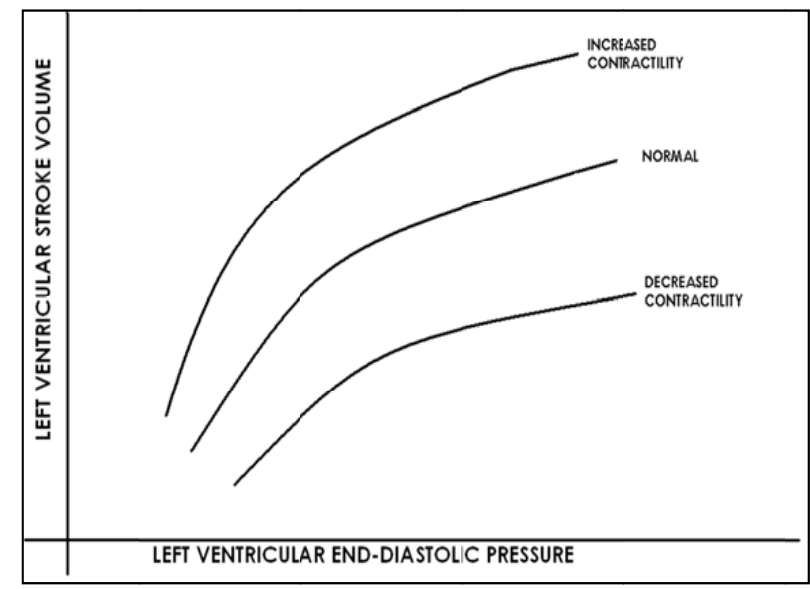

Fig. (1). Frank-Starling relationship of preload verses ventricular function (cardiac output). In the normal heart, increased preload produces improved ventricular function. In the failing heart, the curve is shifted downward and to the right. Inotropic therapy shifts the curve upward and to the left.

\section{SIGNS AND SYMPTOMS}

Infants and children rely on tachycardia as the preferred mechanism to increase cardiac output in a shock state [5]. Therefore, tachycardia is a sensitive early finding, though not specific to cardiogenic shock. In fact, it is not specific to shock at all. Heart rate elevation can be seen in fever, thyroid dysfunction, pain, and a multitude of other conditions. Grady et al. provide a very nice conceptual overview for the rapid assessment of the failing heart - differentiating those patients with adequate perfusion and those with critical hypoperfusion. The adequate perfusion group is further classified as "warm and dry" or "warm and wet" differentiating the extent of pulmonary/systemic vascular congestion despite maintained perfusion. The critical hypoperfusion group consists of "cold and dry" and "cold and wet" patients - the worst of these being the low cardiac output without vascular congestion ("cold and dry") [6]. Clinically these categories may demonstrate themselves in ways other than tachycardia - such as oliguria, changes in skin temperature, and impaired mental status are all due to the preferential shunting of blood flow from end organs in an attempt to optimize "vital" organ perfusion. Clinically, the skin is often described as pale, mottled, cool, and clammy in the shock state [5]. Decreased renal blood flow decreases glomerular filtration rate and thereby decreases urine output. As described above, the renin-angiotensin II-aldosterone system is intimately involved in this process. Impaired mental status is often a late finding and may indicate a shock state that is irreversible. Impaired mentation is a result of decreased cerebral perfusion pressure as blood pressure and subsequently, delivery of energy substrate, decreases [7].

Other physical exam findings consistent with the diagnosis of cardiogenic shock are tachypnea, an S3 gallop, wheezing or rales, dyspnea or cough, cyanosis, diaphoresis, hepatomegaly, jugular vein distention (JVD), and peripheral edema. Most consider hepatomegaly a highly useful exam finding for the pediatric patient presenting in shock - as hepatomegaly may suggest elevated right atrial pressures, a consequence of elevated right ventricular end diastolic pressure/volume. Septic and hypovolemic shock rarely present with excessive right atrial volume/pressure. JVD similarly indicates elevated right atrial volume/pressure although as most pediatric patients have short and stout necks, making the detection of JVD more difficult in this population. Similarly, peripheral edema is often subtle in infants and children, making it a less sensitive exam finding. Biventricular or left-sided cardiac compromise will elevate the left ventricular end-diastolic volume/pressure and cause pulmonary venous congestion. Clinically a child with pulmonary venous congestion will present with crackles and tachypnea. A third heart sound - or S3 gallop - is a result of the rapid filling of the ventricle. Best heard with the bell of a stethoscope just after the second heart sound, the gallop indicates left ventricular dysfunction when auscultated at the apex and right ventricular dysfunction when auscultated at the left lower sternal border. It is highly indicative of a volume overload state and is often followed by a diastolic murmur indicating increased blood flow across the valve. Hypotension is a late and often ominous finding. Definitions of hypotension in pediatric population can be found in Table 1. Even after a patient exhibits signs and symptoms of decreased cardiac output, narrowing the diagnosis to a cardiogenic etiology and initiating the appropriate management for cardiogenic shock can remain challenging because of its myriad of etiologies.

Table 1. Definition of Hypotension Based on Age [5,43]

\begin{tabular}{|c|c|}
\hline Age & Systolic Blood Pressure (mmHg) \\
\hline \hline $0-28$ days & $<60^{\dagger}$ \\
\hline 1 month-12 months & $<70$ \\
\hline $1-10$ years & $<70+(2 x$ age in years $)$ \\
\hline$>10$ years & $<90$ \\
\hline
\end{tabular}

Parameters for normal blood pressure and hypotension in this age group are variable and influenced by post-conceptional age. For example, a normotensive term newborn may have a systolic blood pressure of $40 \mathrm{mmHg}$.

\section{ETIOLOGY}

There are a multitude of causes for cardiogenic shock in infancy and childhood. Dysrhythmias, cardiomyopathy and/or carditis, congenital heart disease and obstruction are four broad categories that can reveal a myriad of causes for cardiogenic shock.

\section{Congenital Heart Lesions}

The main sources of cardiogenic shock that are considered congenital in nature include left ventricular outflow tract obstruction, large left-to-right shunts, and 
physiologic or pathologic pulmonary steal. Viewed as the most common cause of cardiogenic shock in pediatrics, the substrate for congenital heart lesions is not a normal heart and therefore surgical intervention for palliation is often inevitable [8].

Hypoplastic left heart syndrome, critical aortic stenosis, coarctation of the aorta, and hypertrophic cardiomyopathy (HCM) are all culprits in left ventricular outflow tract obstruction (LVOTO). These particular disorders are also discussed in the review on obstructive shock, as the pathophysiology is very similar. All except HCM are ductaldependant lesions that, if not diagnosed prenatally, present in the first week to ten days of life as the patent ductus arteriosus is closing, thereby cutting off systemic blood supply [5]. Prentatal diagnosis is ideal for early initiation of therapy, however a recent study in Australia found it had no impact on post-operative outcome in the case of hypoplastic left heart syndrome [9].

In large left-to-right shunts, patients find themselves entering a state of congestive heart failure in the early neonatal period as their pulmonary vascular resistance falls. Such lesions as atrial-septal defect, ventricular-septal defect, patent ductus arteriosus, and atrio-ventricular septal defect are included in this category. During fetal life, blood supply is shunted around the lungs as the placenta provides adequate oxygenation and the lungs are fluid-filled. As the child transitions to life outside the womb, the first breath begins a process by which pulmonary vascular resistance decreases at a variable rate. In fetal life, pulmonary vascular resistance is high, but as pulmonary blood flow is needed to oxygenate the blood after delivery, over a period of weeks to months, the vascular resistance falls and blood flow increases to the lungs. In patients with large shunts, the blood chooses the path of least resistance and the lowresistance lung circuit receives preferential blood flow, resulting in pulmonary overcirculation and congestive heart failure, leading to inadequate systemic circulation and ultimately cardiogenic shock $[5,10]$.

\section{Inflammatory Disorders of the Heart}

Infectious myocarditis is probably the most widely studied cause for cardiogenic shock in this subset, although other causes range from metabolic, toxic, autoimmune/connective tissue derangements, neuromuscular disorders, hypoxic-ischemic events, idiopathic conditions, or inherited cardiomyopathies. Metabolic causes of shock can include hypo- or hyperthyroidism, hypoglycemia, pheochromocytoma, glycogen storage diseases (i.e. Pompe disease), mucopolysaccharidoses, carnitine deficiency, Fabry disease, disorders of fatty acid metabolism, acidosis, hypothermia, and hypocalcaemia. Such metabolic disorders may cause cardiac dysfunction and eventually failure by either hypertrophic (volume-overload) or dilated (pressureoverload) mechanisms with or without resulting cardiac conduction defects [11].

Myocarditis often presents a challenge to the emergency physician or primary care physician because symptoms can range from low-grade fever to shock. A major cause of sudden death in adults younger than 40 and young athletes, myocarditis is an insidious disease that can often be asymptomatic. Probably underdiagnosed, approximately $20 \%$ of young subjects with sudden cardiac death have histological evidence of myocarditis at autopsy. Early recognition and diagnosis is essential in preventing longterm complications of myocarditis. The current goldstandard in diagnosis remains endomyocardial biopsy which can be "operator-dependant." Key causes include infectious (summarized in Table 2), idiopathic, autoimmune, allergic, and toxic [12].

Toxic reactions to medications such as sulfonamides, penicillins, and anthracyclines can cause cardiomyopathy and subsequent shock. Anthracyclines cause a dilated cardiomyopathy that has long been described in the oncology literature [13]. The mechanism by which penicillins and sulfonamides is not as clear, but is usually associated with an anaphylactoid reaction [14].

Connective tissue diseases such as systemic lupus erythamatosus (SLE), juvenile rheumatoid arthritis (JRE), polyarteritis nodosa, Kawasaki disease, and acute rheumatic fever have also been implicated in causing cardiomyopathy leading to shock or heart failure. Cardiac involvement in lupus can range from pericarditis, myocarditis, endocarditis, and even coronary artery involvement [15].

Neuromuscular disorders known to cause cardiomyopathy include Duchene's muscular dystrophy, myotonic dystrophy, limb-girdle dystrophy, spinal muscular atrophy, and Friedreich's ataxia. Heart failure is a classic complication of muscular dystrophy due to the absence of a key sarcolemmal protein, dystrophin [16]. Myotonic dystrophy and cardiac failure primarily stems from increased risk of conduction disorders, namely first degree heart block and tachyarrythmias. Patients with limb-girdle dystrophy and spinal muscular atrophy suffer from dilated cardiomyopathy $[17,18]$. Onefifth of patients who suffer from Friedreich's ataxia are clinically affected by hypertrophic cardiomyopathy [19]. Their symptomatology can range from asymptomatic to mild disease with palpitations and shortness of breath or may result in sudden cardiac death [20, 21]. Hypoxic-ischemic events leading to cardiac failure range from cardiac arrest and cardiopulmonary bypass to head injury, prolonged shock, or even carbon monoxide poisoning [22].

\section{Dysrhythmias}

Any abnormal rhythm can tax the heart beyond its capacity, inducing cardiogenic shock, heart failure or even sudden death. Mentioned most commonly in the literature are prolonged, unrecognized supraventricular tachycardia (SVT), bradyarythmias, complete heart block, tachyarrythmias such as ventricular tachycardia (with or without pulses) or ventricular fibrillation, and other conditions that could predispose a patient to developing a dysrhythmia such as hypothermia and drug intoxications [23]. Prolonged, unrecognized SVT decreases cardiac output by inadequate relaxation periods in each cardiac cycle and decreased filling time in the atria, thereby decreasing filling pressure in the ventricles. The ventricular tachyarrythmias cause heart failure by both diminishing ventricular filling time and eliminating atrio-ventricular synchrony. The combination results in decreased ventricular filling and compromised stroke volume. 
Table 2. Infectious Myocarditis [12, 44]

\begin{tabular}{|c|c|c|}
\hline Viral & Parasitic & Bacterial \\
\hline \hline Enteroviruses (esp. Coxsackie B) & Trypanosoma cruzi & Chlamydophila psittaci \\
\hline Adenovirus & Trypanosoma brucei gambiense & Corynebacterium diphtheriae \\
\hline Influenza & Trypanosoma brucei rhodesiense & Borrelia spp. \\
\hline Ebstein-Barr & Toxocara canis/cati & Mycoplasma pneumoniae \\
\hline Parvovirus & Toxoplasma gondii & Streptobacillus monoliformis/minus \\
\hline Mumps & Trichinella spiralis \\
\hline West Nile Virus & & \\
\hline HHV-6 & & \\
\hline HIV & & \\
\hline CMV & & \\
\hline HSV-2 & & \\
\hline Hepatitis C & & \\
\hline
\end{tabular}

\section{Obstructive Lesions}

Cardiac tamponade involves a decrease in both right and left ventricular volumes, with normal systolic function. Due to the accumulation in the pericardial sac, the intrapericardial pressure prevents adequate atrial filling due to diastolic atrial compression and thus impaired ventricular filling from the absence of an atrial volume transported to the ventricle. Subsequently, cardiac output and stroke volume decrease [7].

Acute severe pulmonary embolus involving one or both the proximal pulmonary artery may present clinically as cardiogenic shock. Right ventricular ejection fraction is acutely decreased due to the obstruction upstream in the pulmonary artery bifurcation or proximal branch pulmonary arteries. Volume overload with right ventricular overdistension occurs thereby causing a decrease in volume to the left ventricle due to compensatory shifting of the intraventricular septum. The resultant "pancaked" left ventricle is incapable of sustaining meaningful cardiac output [7].

\section{Other Disorders}

Acute valvular regurgitation, namely mitral and aortic, is another cause for cardiogenic shock. Acute mitral regurgitation from traumatic rupture of the chordae or following bacterial endocarditis markedly reduces left ventricular stroke volume thereby increasing ventricular and regurgitant volume. Acute aortic regurgitation is less common in pediatrics and usually caused by infective endocarditis, aortic dissection, or trauma. Faced with a sudden increase in left ventricular diastolic volume due to aortic "backflow" into the left ventricle, stroke volume and cardiac output decrease [7].

Trauma cannot be left out of the discussion on cardiogenic shock, either. Commotio cordis and contusion cordis are two conditions caused by high-velocity deceleration injuries such as a motor vehicle accident or in high-intensity contact sporting events. Damage to the myocardium can cause ventricular arrhythmias, cardiac failure and event sudden cardiac death. Most patients (81$95 \%$ ) will present within 48 hours of their injury and often have other injuries such as flail chest, sternal fracture, or chest wall ecchymosis [12].

\section{DIAGNOSTIC EVALUATION}

After stabilizing a patient in cardiogenic shock according to Pediatric Advanced Life Support (PALS) or other clinical supportive guidelines, the next step would be laboratory evaluation. Typical labs that may be ordered include arterial blood gas, electrolyte profile including ionized calcium, measurement of BUN and creatinine, liver function tests, complete blood count, serum lactate, and occasionally cardiac enzymes. Electrolytes, renal function values, and liver function tests help reveal the adequacy of end-organ perfusion. Complete blood count will reveal anemia that can be worsening the clinical picture through impaired oxygencarrying capacity. Serum lactate and arterial blood gas will reveal the degree of acidosis. Lactic acidosis is due to the transition to anaerobic metabolism because of inadequate delivery of oxygen and decreased clearance of lactate by the liver, kidneys, and skeletal muscle. Mixed venous oxygen saturation or superior vena cava oxygen saturation can be used as surrogates for estimating the effectiveness of oxygen delivery using the Fick Principle [5].

B-type natriuretic protein (BNP) is a well-studied diagnostic test in diagnosing early heart disease in adults. Recently, its use in diagnosing congenital heart disease and heart failure, monitoring post-operative hemodynamics in cardiac surgery patients, progression of disease in cardiomyopathy, and even post-transplant monitoring has increased [24]. BNP levels are significantly increased in all patients with ventricular dysfunction with right ventricular dysfunction more elevated than left. It may also be useful in early diagnosis of allograft rejection in cardiac transplantation. It is a sensitive (81-89\%) and specific (84$97 \%$ ) measure of ventricular dysfunction and levels may be elevated as early as symptoms arise. It can be rapidly performed at the bedside but with a half-life of only 20 minutes, laboratory values may be difficult to interpret [25]. 
Electrocardiogram and chest radiography are noninvasive tests that may clarify the etiology and severity of cardiac failure. Chest radiograph may demonstrate cardiomegaly, pulmonary venous congestion, or both. Electrocardiogram (ECG) can reveal ventricular hypertrophy, ischemia, or can even be normal [7].

One of the next steps in treating cardiogenic shock is a visual assessment of cardiac structure and function. Echocardiogram can be performed at the bedside to emergently determine structure and systolic function, but less effectively diastolic function. Ejection fraction, wall dynamics, ventricular size and wall thickness, and valvular regurgitation are just a few of the details that will provide insight into cardiac function. Another value that is calculated by either the echocardiographer is the myocardial performance index (MPI) (Eq.2). MPI is a simple, reproducible and non-invasive measure of combined systolic and diastolic ventricular function. MPI is a comparison of the isovolumetric contraction (ICT) and relaxation times (IRT) to the ventricular ejection time (VET).

\section{MPI $=($ Isovolumetric Contraction Time + Isovolumetric Relaxation Time)/ \\ Ventricular Ejection Time}

As systolic function worsens, ICT lengthens and VET shortens, MPI is increased. With worsened diastolic function, IRT lengthens and MPI is similarly increased. A benefit of MPI is that it is not limited by geometric shape of the ventricle [26].

\section{MANAGEMENT}

First line treatment in any form of shock involves stabilizing airway, breathing and circulation. Oxygen supplementation, stabilization of the airway and establishment of vascular access are necessities. There are, however, some conditions that oxygen can be detrimental to the patient. Oxygen is a pulmonary vasodilator, so in singleventricle physiology, dilating the pulmonary vascular bed can worsen pulmonary overcirculation and systemic "steal." [27].

Goal-directed therapy is a bundle of care including intensive care monitoring, fluids, blood products, inotropy and rapid turn-around time in laboratory evaluation in maximizing patient care [28]. Goal-directed therapy is known in the intensive care literature to improve patient outcome [29]. One unit of measurement is the mixed venous oxygen content, which has been used to assess level of tissue oxygenation and has shown a correlation with cardiac output in critically ill patients. A true mixed venous oxygen saturation is a measurement of the oxygen content of the pulmonary artery and is rarely measured directly unless a pulmonary arterial catheter is in place. Instead, we frequently measure the oxygen content from the superior vena cava, which correlates with true mixed venous saturation. Patients with cardiogenic shock had low mixed venous saturations and patients with relatively high levels had better prognosis [30]. Goal-directed therapy is extensively studied in the cardiac intensive care where inadequate oxygenation postoperatively is associated with increased morbidity and mortality [28].
Another important feature in treatment of cardiogenic shock in its acute phase is judicious use of fluid resuscitation. As mentioned above, patients can present hypo-, hyper-, or euvolemic and in cardiogenic shock that is can be difficult to differentiate. The initial management for any form of shock is a fluid challenge. Typical fluid resuscitation is a crystalloid such as lactated ringers or normal saline and the starting dose is $20 \mathrm{ml} / \mathrm{kg}$ given as fast as possible. If the patient is suffering from cardiogenic shock, fluid resuscitation will offer no improvement and may even worsen patient status [31].

\section{Advanced Therapeutic Measures}

There are several principles in the advanced management of severe heart failure or cardiogenic shock. First, it is ideal to minimize myocardial oxygen demand. This is done by intubating and mechanically ventilating the patient and taking away the work and tissue demand associated with an increased work of breathing. Normothermia is also ideal in minimizing oxygen demand. Aggressive use of anti-pyretics or heating apparatuses is of utmost importance. Sedation or even paralysis of the patient eliminates movement of skeletal muscle as a source of oxygen consumption, as well. The next step in medical management of cardiogenic shock is aimed at maximizing myocardial performance. Correction of any dysrhythmia, optimizing preload, improving contractility, and reducing afterload are all staples in maximizing cardiac performance. Preload is optimized with the use of diuretics, vasodilators, and aggressive fluid management (depending on the clinical assessment of volume status). Contractility is improved by correction of metabolic derangements including adequate serum ionized calcium - and providing inotropic support. Afterload reduction is accomplished by providing sedation and pain control and by using the appropriate pharmacologic agents. Table 3 outlines common medications used for hemodynamic and inotropic support. In left-ventricular outflow tract obstruction where systemic blood flow is dependent on the patency of the ductus, Prostaglandin is first-line in medical management. Starting dose is $0.03 \mathrm{mcg} / \mathrm{kg} / \mathrm{min}$ and dose is titrated to response. Doses as high as $0.05-0.1 \mathrm{mcg} / \mathrm{kg} / \mathrm{min}$ have been used but can be associated with significant hypotension and apnea [26].

Afterload reduction can be provided by either Nitroprusside, or more commonly, Milrinone. Nitroprusside is a vasodilator, acting mainly on arterial smooth muscle, but to some extent the venous system as well. Usual dosing for Nitroprussside is $0.5-10 \mathrm{mcg} / \mathrm{kg} / \mathrm{min}$. Milrinone is a type III phosphodiesterase inhibitor that increases cardiac contractility by elevating the intracellular cyclic adenosine monophosphate (cAMP) concentration through inhibiting its degradation. Milrinone also helps to improve diastolic dysfunction and causes some vasodilatation, but does not affect myocardial oxygen consumption [32]. Milrinone is truly the workhorse of cardiogenic shock - and has accurately been described as an inodilator. Other staples in the medical management of cardiogenic shock include the correction of metabolic acidosis or other electrolyte abnormalities, transfusion of blood products (if anemic), or even administration of Vitamin K, cryoprecipitate or fresh frozen plasma if coagulation defects are present [28]. 
Table 3. Vasoactive Medications [5]

\begin{tabular}{|c|c|c|c|}
\hline Agent & Dose & Site of Action & Clinical Effect \\
\hline \hline Dopamine & $3-20 \mathrm{mcg} / \mathrm{kg} / \mathrm{min}$ & $\begin{array}{c}\text { Beta 1; increasing Alpha } \\
\text { effect with increasing dose }\end{array}$ & Increases contractility, vasoconstricts, increases HR, increases PVR \\
\hline Dobutamine & $1-20 \mathrm{mcg} / \mathrm{kg} / \mathrm{min}$ & Beta $2>$ Beta 1 & Increases contractility, vasodilates (Beta2), decreases PVR \\
\hline Epinephrine & $0.01-1 \mathrm{mcg} / \mathrm{kg} / \mathrm{min}$ & Beta $>$ Alpha & Increases contractility and HR, vasoconstricts \\
\hline Norepinephrine & $0.01-1 \mathrm{mcg} / \mathrm{kg} / \mathrm{min}$ & Alpha $>$ Beta & Vasoconstricts, increases SVR, increases contractility and HR \\
\hline Phenylephrine & $0.1-0.5 \mathrm{mcg} / \mathrm{kg} / \mathrm{min}$ & Alpha & Vasoconstricts, increases SVR \\
\hline Vasopressin & $0.0003-0.008 \mathrm{U} / \mathrm{kg} / \mathrm{min}$ & V1 vascular receptor & Vasoconstricts, vasodilates Circle of Willis, stimulates cortisol secretion \\
\hline
\end{tabular}

When medical management is not enough, the next step is intervention of some nature. Current options include extracorporeal membrane oxygenation (ECMO), ventricular assist devices (VAD), and lastly cardiac transplantation. In any case, invasive support - such as ECMO and VAD are often thought of as bridges to the next step. Table 4 outlines the indications for mechanical support in children.

Table 4. Indications for Mechanical Support in Children Adapted from Deiwick et al. [34]

\begin{tabular}{|ll|l|}
\hline \multicolumn{1}{|c|}{ Bridge } & \multicolumn{1}{c|}{ Goal } \\
\hline \hline - & To Treatment & $\begin{array}{l}\text { Stabilize organ function prior to diagnosis } \\
\text { and/or surgery }\end{array}$ \\
\hline - & To Transplant & Support until donor heart is available \\
\hline - To Recovery & $\begin{array}{l}\text { Support until native heart recovers, } \\
\text { no need for transplant }\end{array}$ \\
\hline - To Long-term Bridge & $\begin{array}{l}\text { Support until switch from short-term device } \\
\text { to long-term device is feasible }\end{array}$ \\
\hline
\end{tabular}

\section{Extracorporeal Membrane Oxygenation (ECMO)}

ECMO is a complex system designed to circumvent the lungs and heart in cases of severe cardiac and/or pulmonary failure. ECMO can be used for 2 broad categories of patients: refractory hypoxemic respiratory failure or refractory circulatory failure (the focus for our discussion). It is currently the most commonly used mechanical support system for infants and young children and has distinct advantages over other support mechanisms [33]. An outline of advantages and disadvantages of ECMO can be found in Table 5, though the common significant complications with ECMO are hemorrhagic complications associated with the requirement for anti-coagulation and organ failure related to the non-physiologic, non-pulsatile arterial flow patterns [34].

There are currently several modes of ECMO available, but the most commonly used support method for cardiac failure and cardiogenic shock is the venoarterial (VA) ECMO. In VA ECMO, the right atrium is drained by venous cannulation, allowing desaturated venous blood to be removed from the body. This desaturated blood is pumped to the oxygenator where gas exchange occurs. Gas exchange in the membrane oxygenator depends on the permeability of the membrane to oxygen and carbon dioxide, the available surface area, the pressure gradient, and the interface time.
Viscosity, temperature, and $\mathrm{pH}$ of the blood can also affect gas exchange. After oxygenization, the oxygenated blood is rewarmed to body temperature and returned to the patient via an arterial cannula. Because the adequacy of flow depends upon the volume of deoxygenated blood removed, venous return must be maintained for ECMO to be effective. When myocardial function is severely impaired left atrial decompression may be required, and is often accomplished via atrial septostectomy. Without left atrial decompression, pulmonary edema will develop and recovery of left ventricular function will be impaired. ECMO circuit resistance can be met at the cannula site, so a large bore cannula is ideal. Any process that limits right atrial filling, such as a pneumothorax or pneumopericardium will also hinder venous drainage. Similar problems can occur on the arterial side.

Table 5. ECMO at A Glance

Adapted from Deiwick et al. [34]

\begin{tabular}{|c|c|}
\hline Advantages & Disadvantages \\
\hline \hline Simple cannulation technique & Duration of support \\
\hline Peripheral cannulation & Oxygenator may fail \\
\hline Cost reduction & Need for trained personnel \\
\hline Readily available & Bleeding complications \\
\hline Biventricular support & Thromboembolic events \\
\hline Reliable oxygenation & Immobility \\
\hline & Non-pulsatile flow \\
\hline
\end{tabular}

Less commonly, venovenous (VV) ECMO can be used, employing only central venous access and no central arterial access. Candidates for VV ECMO must have isolated hypoxemic respiratory failure with largely preserved cardiac function. Neonates with congenital diaphragmatic hernia had similar outcomes for VA and VV ECMO [35]. but in patients with cardiac failure VA ECMO is the norm.

Many authors suggest that ECMO seems to be superior to other modes of support in cases of complex congenital heart disease where hypoxia, pulmonary hypertension, or biventricular failure all contribute to the need for support [34]. ECMO is effective at bridging nearly half of eligible children to transplant [33]. Though ECMO has enjoyed gains in survival, most agree that opportunity for improvement 
exists either by modifying ECMO support regimens or by employing alternative extracorporeal devices.

\section{Ventricular Assist Devices}

Ventricular assist devices (VADs) are mechanical pumps that take over the function of one or both ventricles in an attempt to restore normal hemodynamics and end-organ blood flow [34]. Until recently, VADs were used only in adults and older children. Currently, however, centrifugal pump and pulsatile VADs have grown in popularity for pediatric support and have been used in children weighing less than $6 \mathrm{~kg}$ [36]. A summary of VADs can be found in Table 6.

Table 6. VAD at a Glance [31-35]

\begin{tabular}{|c|c|}
\hline Advantages & Disadvantages \\
\hline - Direct Decompression of LV & $\begin{array}{l}\text { - Requires direct cardiac } \\
\text { cannulation }\end{array}$ \\
\hline - Pulsatile Blood Flow & $\begin{array}{l}\text { - } \\
\text { nequires and increased } \\
\text { number of cannulation sites }\end{array}$ \\
\hline $\begin{array}{l}\text { Decreased risk for } \\
\text { hemorrhagic complications }\end{array}$ & $\begin{array}{l}\text { - Unable to use with co- } \\
\text { existing pulmonary } \\
\text { hypertension }\end{array}$ \\
\hline $\begin{array}{l}\text { - Decreased need for blood } \\
\text { products }\end{array}$ & $\begin{array}{l}\text { - Respiratory failure must not } \\
\text { be present to use }\end{array}$ \\
\hline $\begin{array}{l}\text { - Decrease in level of systemic } \\
\text { anti-coagulation }\end{array}$ & $\begin{array}{l}\text { - Only useful in single } \\
\text { ventricle failure }\end{array}$ \\
\hline - Mobility & $\begin{array}{l}\text { - Cannot be used in presence } \\
\text { of intracardiac shunt }\end{array}$ \\
\hline $\begin{array}{l}\text { Ability to extubate, feed } \\
\text { enterally, even discharge home }\end{array}$ & \\
\hline
\end{tabular}

There are advantages to VAD use over the use of ECMO. VADs allow for direct decompression of the left ventricle and can provide pulsatile blood flow [33]. VADs do not require an oxygenator in circuit, therefore trauma to blood elements is reduced, which decreases the requirement for excessive transfusion and the development of sensitivity to HLA surface antigen. In addition, VADs allow a decreased dose of systemic anticoagulation and thereby reducing the risk of hemorrhagic complications, which are well-known with ECMO. In a retrospective review, McMullen et al. describe a cohort of two similar patient populations in terms of age, gender, and body surface area who received either ECMO or VAD via a centrifugal pump and found that outcome, recovery time, and circuit complications were similar but patients on VAD therapy had significantly less blood product administration and hemorrhage in the postoperative period. Patients on ECMO therapy required higher doses of anti-coagulants and higher activated clotting times to maintain patency of circuit, as well $[33,37]$.

VAD support is best used when isolated left ventricular failure is the only indication for assistance [34]. VADs can be used for a longer amount of time than ECMO, as well. A recent study at the Cleveland Clinic noted that ECMO support was limited to two weeks due to complications from non-pulsatile flow, whereas VAD use was as long as 98 days [36]. However, it is suggested that after 72 hours of mechanical ECMO support without recovery of myocardial function, a decision should be made concerning transplant, longer-term assist device implementation, or termination of support [34]. A secondary advantage of VAD support may be related to the "resting of the heart" which has been shown to demonstrate restoration of the more normal protein structures (collagen and dystrophin) as well as reduction in myocardial hypertrophy [38].

A distinct disadvantage of VAD support is that it requires direct cannulation of the heart via sternotomy and requires 4 cannulation sites compared to ECMO's 2 cannulation sites [36]. There are also some contraindications to the use of VAD for mechanical support. Pulmonary hypertension, respiratory failure, biventricular cardiac failure, and residual intracardiac shunts are all relative contraindications for VAD support [34].

Paracorporeal VADs are also available for use in the pediatric population to a limited extent. The benefit of this system is that child is able to be extubated and can mobilize while awaiting transplantation. However, the device is currently not approved for use by the FDA and must only be used on the basis of "compassionate use." [39]. It is clear that the technology and experience associated with VAD use in the pediatric age group is evolving at a rapid pace, providing reasonable expectation that the next decade will provide much more clarity on this relatively novel pediatric therapeutic option.

\section{Transplantation}

Heart transplantation is the last therapeutic option for children suffering from terminal heart failure refractory to medical therapy and often dependant on mechanical supportive interventions [40]. One of the major drawbacks of pediatric heart transplant is the lack of a suitable donor pool due to the size restrictions of a small child's thoracic cavity. For this reason, patients with dilated cardiomyopathy seem to fare better in the selection process because of the large size of their native heart. Prognosis for pediatric patients awaiting transplant is guarded, at best. Short-term mortality while awaiting a compatible organ can be up to $20 \%$ overall for children and as high as $31 \%$ in young children (less than 6 months) [41]. Increased pediatric pre-transplant mortality coupled with poor donor pool warrants better pre-transplant therapies in the form of pediatric-sized ventricular assist devices. In 1995 the Stanford group reported a $1 \mathrm{yr}$ survival of $75 \%$ and $5 \mathrm{yr}$ survival of $60 \%$ after transplant for treatment of end-stage heart failure. More recently, however survival data are more reassuring. One-year survival was $94.6 \%$ and 5 -year survival was $86.1 \%$ in a British cohort. High pre-transplant pulmonary vascular resistance, right ventricular failure and restrictive cardiomyopathy were poor prognostic factors for survival after transplant. Long-term follow-up acute rejection and infection were the most common sources of mortality [42].

\section{OUTCOME}

Due to the variety of causes of cardiogenic shock in children, outcome is highly variable and dependant on both the etiology and treatment. The vast majority of patients presenting with cardiogenic shock are successfully managed with either conventional cardio-respiratory support techniques and medications or support and subsequent 
correction of the anatomic cardiac defect. The outcomes of these patients depend on the nature of the myocardial inflammation and injury and the reversibility (either medically or surgically) or the myocardial dysfunction. There are, however, patients that progress to myocardial failure requiring support devices. Poor neurologic outcome was more common with ECMO as recently as 2002. More than $60 \%$ of ECMO patients demonstrated moderate to severe impairment at 4 year follow-up. Only $20 \%$ of patients supported with VAD showed the same impairment [36]. Intracranial hemorrhage and thromboembolic cerebral events occur in 3-6\% of pts regardless of mode of support [34]. One study showed that children with congestive cardiac failure fared poorly when treated with VAD. Fewer than 33\% survived to transplantation [33]. Myocarditis has the highest survival of any patient population requiring ECMO with $58 \%$ of patients able to be weaned from support. A recent study proved an $80 \%$ survival in patients with myocarditis supported by either ECMO or VAD [36]. Experimental use of Paracorporeal VAD has successfully bridged patients to transplant $60 \%$ of the time [39]. Transplant outcomes remain "good," though organ availability remains limited.

\section{CONCLUSION}

Cardiogenic shock often provides a clinically challenging diagnostic dilemma for the clinician caring for the infant with shock. However, cardiogenic shock should neither be ignored nor forgotten as solving its puzzle, for most practitioners, warrants a careful understanding of the complex physiologic sequelae and the variety of inciting causes. The most common causes remain congenital heart defects and infectious myocarditis. Fortunately, it seems that the growing trend toward diagnosing congenital heart defects prenatally may reduce the burden of undiagnosed structural heart defects and resultant cardiogenic shock, allowing for earlier treatment and an increased survival. Unfortunately, the same advances have not been made in terms of diagnosing infectious myocarditis, causing the medical world to rely largely on clinical judgment. Symptomatology of cardiogenic shock greatly overlaps both other types of shock as well as non-shock states, however ancillary testing such as echocardiography is diagnostic of the myocardial dysfunction. Unfortunately due to the fact that the cardiovascular system is more times than not involved in all forms of shock, early recognition of shock is key to preventing irreversible myocardial damage. Treatment mechanisms such ECMO and VADs allow practitioners to provide a bridge to recovery, surgical correction or transplant. Cardiac transplant outcome in children is improving, but lack of an adequate donor pool still exists as a limiting factor to its use. Specialty care through a pediatric cardiologist and often a pediatric cardiothoracic surgeon should be sought early to prevent the irreversible damage that can often end a child's life.

\section{CONFLICT OF INTEREST}

The authors confirm that this article content has no conflicts of interest.

\section{ACKNOWLEDGEMENTS}

Declared none.

\section{REFERENCES}

[1] Court O, Kumar A, Parrillo J. Clinical review: Myocardial depression in sepsis and septic shock. Crit Care 2002; 6(6): 500-8.

[2] Domico M, Liao P, Anas N, Mink R. Elevation of brain natriuretic peptide levels in children with septic shock. Pediatr Crit Care Med 2008; 9(5): 478-83.

[3] Cox DJ, Groves AM. Inotropes in preterm infants--evidence for and against. Acta Paediatr Suppl 2012; 101(464): 17-23.

[4] Noori S, Seri I. Neonatal blood pressure support: the use of inotropes, lusitropes, and other vasopressor agents. Clin Perinatol 2012; 39(1): 221-38.

[5] McKiernan CA, Lieberman SA. Circulatory shock in children: an overview. Pediatr Rev 2005; 6(12): 451-60.

[6] Grady K, Dracup K, Kennedy G, et al. Team management of patients with heart failure: A statement for healthcare professionals from The Cardiovascular Nursing Council of the American Heart Association. Circulation 2000; 102(19): 2443-56.

[7] Chatterjee K, McGlothlin D, Michaels A. Analytic reviews: cardiogenic shock with preserved systolic function: a reminder. J Intensive Care Med 2008; 23(6): 355-66.

[8] Radermecker M, Canivet JL, Lancellotti P, Limet R. The usual causes of left ventricular outflow tract obstruction below the aortic valve in normal ventriculoarterial connection: review of the physiopathology and surgical implications. Acta Chir Belg 2005; 105(5): 475-81.

[9] Sivarajan V, Penny DJ, Filan P, Brizard C, Shekerdemian LS. Impact of antenatal diagnosis of hypoplastic left heart syndrome on the clinical presentation and surgical outcomes: the Australian experience. J Paediatr Child Health 2009; 45(3): 112-7.

[10] Mann D, Qu JZ, Mehta V. Congenital heart diseases with left-toright shunts. Int Anesthesiol Clin 2004; 42(4): 45-58.

[11] Kane JM, Rossi J, Tsao S, Burton BK. Metabolic cardiomyopathy and mitochondrial disorders in the pediatric intensive care unit. $\mathrm{J}$ Pediatr 2007; 151(5): 538-41.

[12] Hsing DD, Madikians A. True-true, unrelated: a case report. Pediatr Emerg Care 2005; 21(11): 755-9.

[13] Rathe M, Carlsen NL, Oxhoj H, Nielsen G. Long-term cardiac follow-up of children treated with anthracycline doses of 300 $\mathrm{mg} / \mathrm{m}(2)$ or less for acute lymphoblastic leukemia. Pediatr Blood Cancer 2009; 54(3): 444-8.

[14] Hartmann M, Bode C, Zirlik A. Anaphylactic shock-associated cardiomyopathy. Int J Cardiol 2008; 127(3): e136-7.

[15] Jain D, Halushka MK. Cardiac pathology of systemic lupus erythematosus. J Clin Pathol 2009; 62(7): 584-92.

[16] Fayssoil A, Nardi O, Orlikowski D, Annane D. Cardiomyopathy in Duchenne muscular dystrophy: pathogenesis and therapeutics. Heart Fail Rev 2010; 15(1): 103-7.

[17] Bouhouch R, Elhouari T, Oukerraj L, et al. Management of cardiac involvement in neuromuscular diseases: review. Open Cardiovasc Med J 2008; 2: 93-6.

[18] Distefano G, Sciacca P, Parisi MG, et al. [Heart involvement in progressive spinal muscular atrophy. A review of the literature and case histories in childhood]. Pediatr Med Chir 1994; 16(2): 125-8.

[19] Casazza F, Morpurgo M. The varying evolution of Friedreich's ataxia cardiomyopathy. Am J Cardiol 1996; 77(10):895-8.

[20] Pandolfo M. Friedreich ataxia: the clinical picture. J Neurol 2009; 256 (Suppl 1): 3-8.

[21] Gucev Z, Tasic V, Jancevska A, et al. Friedreich ataxia (FA) associated with diabetes mellitus type 1 and hyperthrophic cardiomyopathy. Bosn J Basic Med Sci 2009; 9(2): 107-10.

[22] Yanir Y, Shupak A, Abramovich A, Reisner SA, Lorber A. Cardiogenic shock complicating acute carbon monoxide poisoning despite neurologic and metabolic recovery. Ann Emerg Med 2002; 40(4): 420-4.

[23] Silva JN, Van Hare G. Management of postoperative pediatric cardiac arrhythmias: current state of the art. Curr Treat Options Cardiovasc Med 2009; 11(5): 410-6.

[24] Das B, Raj S, Solinger R. Natriuretic peptides in cardiovascular diseases of fetus, infants and children. Cardiovasc Hematol Agents Med Chem 2009; 7(1): 43-51.

[25] Das B. Plasma B-type natriuretic peptides in children with cardiovascular diseases. Pediatr Cardiol 2010; 31(8): 1135-45.

[26] Fesseha AK, Eidem BW, Dibardino DJ, et al. Neonates with aortic coarctation and cardiogenic shock: presentation and outcomes. Ann Thorac Surg 2005; 79(5): 1650-5. 
[27] Gupta A, Mogos C, Schmer V, Gudavalli M. One day old infant with acyanotic congenital heart disease: critical aortic stenosis. J Perinat Med 1999; 27(4): 292-4.

[28] Gordon A, Russell J. Goal directed therapy: how long can we wait? Crit Care 2005; 9(6): 647-8.

[29] Rossi A, Khan D, Hannan R, Bolivar J, Zaidenweber M, Burke R. Goal-directed medical therapy and point-of-care testing improve outcomes after congenital heart surgery. Intensive Care Med 2005; 31(1): 98-104

[30] Hainsworth R. Mixed venous oxygen content and its meaning. Intensive Care Med 1981; 7(4): 153-5.

[31] Kaufman BS. Pharmacology of colloids and crystalloids. Crit Care Clin 1992; 8: 235-53.

[32] Joynt C, Bigam DL, Charrois G, Jewell LD, Korbutt G, Cheung PY. Dose-response effects of milrinone on hemodynamics of newborn pigs with hypoxia-reoxygenation. Intensive Care Med 2008; 34(7): 1321-9.

[33] Fynn-Thompson F, Almond C. Pediatric ventricular assist devices. Pediatr Cardiol 2007; 28(2): 149-55.

[34] Deiwick M, Hoffmeier A, Tjan TD, Krasemann T, Schmid C, Scheld HH. Heart failure in children -- mechanical assistance. Thorac Cardiovasc Surg 2005; 53 (Suppl 2): S135-40.

[35] Kugelman A, Gangitano E, Pincros J, Tantivit P, Taschuk R, Durand M. Venovenous versus venoarterial extracorporeal membrane oxygenation in congenital diaphragmatic hernia. J Pediatr Surg 2003; 38(8): 1131-6.

[36] Duncan B. Mechanical circulatory support for infants and children with cardiac disease. Ann Thorac Surg 2002; 73(5): 1670-7.
McMullan D, Emmert J, Permut L, et al. Minimizing bleeding associated with mechanical circulatory support following pediatric heart surgery. Eur J Cardiothorac Surg 2011; 39(3): 392-7.

[38] Vatta M, Stetson S, Perez-Verdia A, et al. Molecular remodelling of dystrophin in patients with end-stage cardiomyopathies and reversal in patients on assistance-device therapy. Lancet 2002; 359(9310): 936-41.

[39] Malaisrie S, Pelletier M, Yun J, et al. Pneumatic paracorporeal ventricular assist device in infants and children: initial Stanford experience. J Heart Lung Transplant 2008; 27(2): 173-7.

[40] Groetzner J, Reichart B, Roemer U, et al. Results of pediatric cardiac transplantation -- long-term results of a 15-year experience. Thorac Cardiovasc Surg 2005; 53 Suppl 2: S149-54.

[41] Jatene M, Miana L, Pessoa A, et al. Pediatric heart transplantation in refractory cardiogenic shock: a critical analysis of feasibility, applicability and results. Arq Bras Cardiol 2008; 90(5): 329-33.

[42] Hoskote A, Carter C, Rees P, Elliott M, Burch M, Brown K. Acute right ventricular failure after pediatric cardiac transplant: predictors and long-term outcome in current era of transplantation medicine. J Thorac Cardiovasc Surg 2010; 139(1): 146-53.

[43] Zubrow AB, Hulman S, Kushner H, Falkner B. Determinants of blood pressure in infants admitted to neonatal intensive care units: a prospective multicenter study. Philadelphia Neonatal Blood Pressure Study Group. J Perinatol 1995; 15(6): 470-9.

[44] Kühl U, Schultheiss H. Viral myocarditis: diagnosis, aetiology an management. Drugs 2009; 69(10): 1287-302.

(c) Smith and Bigham; Licensee Bentham Open.

This is an open access article licensed under the terms of the Creative Commons Attribution Non-Commercial License (http://creativecommons.org/licenses/by$\mathrm{nc} / 3.0 /$ ) which permits unrestricted, non-commercial use, distribution and reproduction in any medium, provided the work is properly cited. 